\title{
COMPUTATIONAL MODELING OF THE EXPERIMENTAL RESPONSE OF MICROSCALE BISTABLE TENSEGRITY STRUCTURES
}

\section{ZACHARIAS VANGELATOS ${ }^{1}$, ANDREA MICHELETTI ${ }^{2}$, NARINDER SINGH $^{3}$, COSTAS P. GRIGOROPOULOS ${ }^{1}$ AND FERNANDO FRATERNALI ${ }^{3}$}

\author{
${ }^{1}$ Department of Mechanical Engineering \\ University of California, Berkeley, CA, USA \\ zacharias_vangelatos@berkeley.edu, cgrigoro@berkeley.edu \\ ${ }^{2}$ Department of Civil and Computer Science Engineering \\ University of Rome Tor Vergata, Italy \\ micheletti@ing.uniroma2.it \\ ${ }^{3}$ Department of Civil Engineering \\ University of Salerno, Italy \\ snarinder@unisa.it, f.fraternali@unisa.it
}

Key words: Architected Metamaterials, Tensegrity Systems, Bistability, Multiphoton Lithography, Indentation testing

\begin{abstract}
We report about the analysis, design, and experimental testing of modular structures composed of bistable units derived from the classic triangular tensegrity prism. Tensegrity structures are pinconnected frameworks, composed by bars and cables, possessing internal mechanisms and self-stress states, and featuring a variety of structural responses depending on their prestress, edge connectivity, and geometry. When a tensegrity system has only one internal mechanism and one self-stress state, as in the triangular prism case, it is possible to associate to it a corresponding bistable unit, by replacing all cables with bars and changing their edge-lengths slightly. After presenting experimental results of compression tests carried out on microscale specimens fabricated through multiphoton lithography, we compare them with the numerical predictions obtained by our computational model.
\end{abstract}

\section{INTRODUCTION}

In recent times, architected metamaterials, structural systems whose physical behavior depends on the way they are designed rather than from the bulk properties of the constituent material, are attracting more and more attention from the scientific community. In particular, metamaterials are designed so as to obtain extraordinary mechanical properties, such as exceptional strength-to-weight and stiffness-toweight ratios, frequency bandgaps, negative overall elastic moduli, negative mass density, auxeticity, and solitary wave propagation [1]-[13]. Bistability is one of the difficult-to-find and most desired features in current studies on architected materials [14]- [19]. Tensegrity systems, pin-connected prestressed frameworks composed by bars and cables, constitute a particular structural class in that their response 
to statical and dynamical actions incude a rich variety of non-linear effects [20,21], [10]-[13]. We here focus on the bistability property of some tensegrity structures [21] to design and fabricate bistable latticed structures at the nanoscale. The fabrication is performed by means of multiphoton lithography [22,23], the only available technique to additively manufacture the lattices we analyze in this study.

This paper is organized as follows. In Section 2, we describe the method to derive bistable lattices from a particular subclass of tensegrity systems. In Section 3 we give details about the fabrication technique and experimental setup, together with the results of the indentation tests. Numerical predictions obtained by a reduced order elastic model are reported in Section 4. Our conclusions follow in Section 5.

\section{BISTABLE LATTICES WITH TENSEGRITY ARCHITECTURE}

Tensegrity systems are characterized by the existence of a self-stress state, that is a set of axial forces in the elements in equilibrium with null external loads, and they can also possess internal mechanisms, which are nodal displacements which cause null first-order elongation of the elements, excluding rigidbody displacements. Self-stress states and mechanisms belongs to the left and right nullspaces of the equilbrium operator, expressing the linear relationship betweeen the axial forces in the elements and the extenal loads [24]. We consider here the subclass of tensegrity systems possessing a single internal mechanism and a single self-stress state, which means that the equilibrium operator is represented by a rank-deficient square matrix, with rank deficiency equal to one. Stable tensegrity systems with internal mechanisms satisfy the so-called prestress-stability condition, signifying that the self-stress state im-
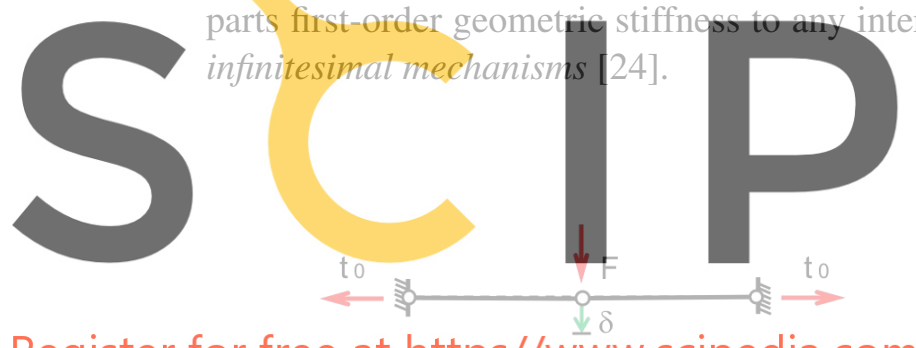

Register for free at https//www.scipedia.com tc

(a)

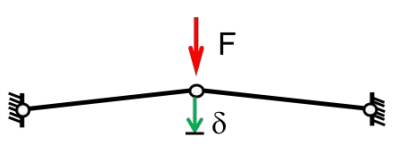

(d)

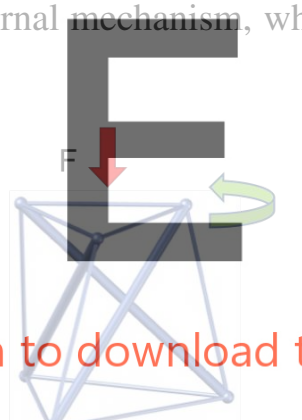

(b)

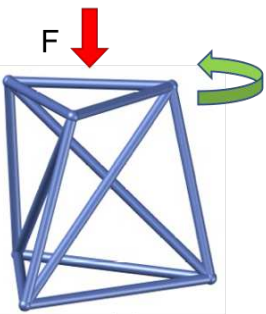

(e)

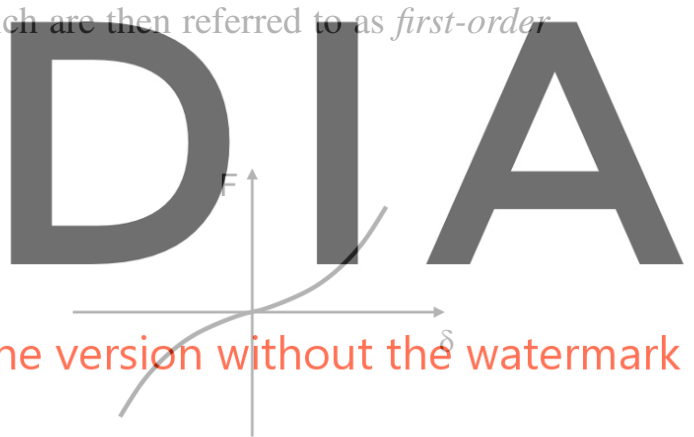

(c)

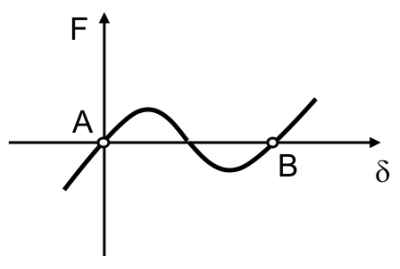

(f)

Figure 1: (a) Three-aligned-hinges system. (b) Triangular tensegrity prism. (c) Monostable load-vs-displacement plot. (d) Simple bistable system. (e) A bistable bar-framework corresponding to the tensegrity prism in (b). (f) Bistable load-vs-displacement plot. Curved green arrows in (b) and (e) indicate the twisting of the top base in the relative screw motion between bases. 
Figure 1 illustrates the behavior of two prestress-stable systems, the simple three-aligned-hinges system (Fig. 1, a) [25], and the classical tensegrty prism (Fig. 1, b), when they are subjected to a load activating the mechanism. The typical load-vs-displacement curve is of stiffening type, and it can be approximated by a cubic with an inflexion point at the origin (Fig. 1, c). The slope at the origin is directly proportional to the level of self-stress $t_{0}$ in the elements. It can be verified that, by reversing the sign of prestress of a tensegrity system with the above-mentioned properties, the equilibrium configuration becomes unstable, and that two other stress-free stable equilibrium configuration arise (Fig. 1, d, e). The response to a load activating the internal mechanism is then of bistable type (Fig. 1, f) and it is associated to a double-well elastic energy.

A triangular tensegrity prism can be obained by considering a triangular prism with nodes at the vertices, bars along the edges, and cables along the diagonals of the lateral faces. In the stable prestressed equilibrium configuration, the top base is rotated with respect to the bottom base by an angle equal to $\theta_{0}=\pi / 6$, which is referred to as the twist angle at equilibrium (cf [20]). This value does not change when considering different circumscribed radii of the two bases, as shown in Fig. 1 (b). The first-order infinitesimal mechanims is a relative screw motion (roto-translation) between bases, with the screw axis passing through the bases' centroids.

The corresponding bistable unit is obtained by replacing cables with bars, and realizing the edge-lengths so as to have a twist angle slightly different from $\pi / 6$. The structure is stress-free in this configuration, which we name as primary stable configuration (corresponding to point A in Fig. 1, f). When applying a vertical load to the top base, while having the bottom base fixed to the ground, the system snaps into the

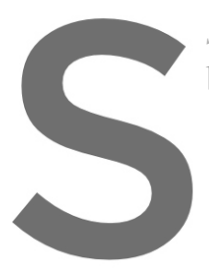
secondary stable configuration between bases, analogous to the
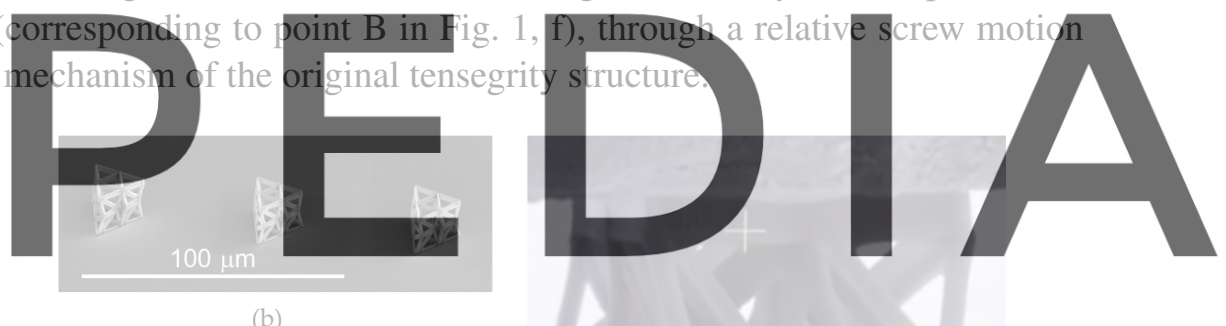

Register for free at https//www.scipedia.com to download the version without the watermark

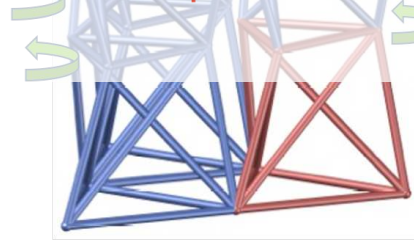

(a)

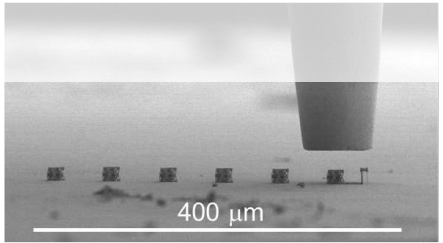

(c)

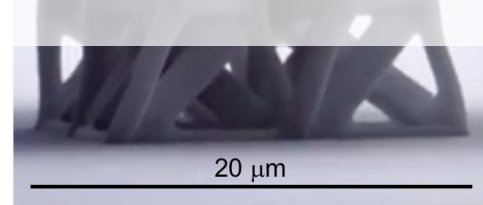

(d)

Figure 2: (a) Bistable assembly composed of six tensegrity prisms (one highlighted in red). (b, c) Fabricated samples. (d) View of a sample during the indentation experiment.

\section{FABRICATION BY MPL AND INDENTATION EXPERIMENTS}

In order to test the bistable behavior of this system, we designed the modular assembly in Fig. 2 (a) where three double units are assembled together side by side, a double unit being composed of two prisms which are the mirror image of each other. The size of the middle bases is chosen so as to prevent 


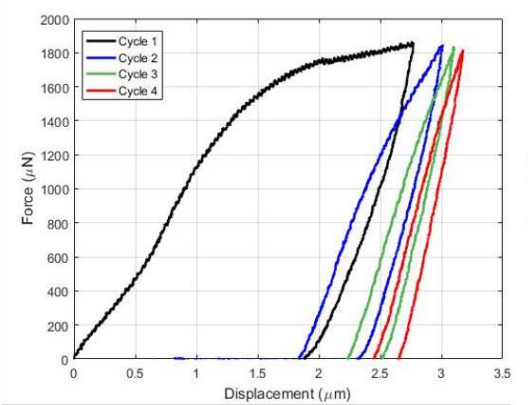

(a)

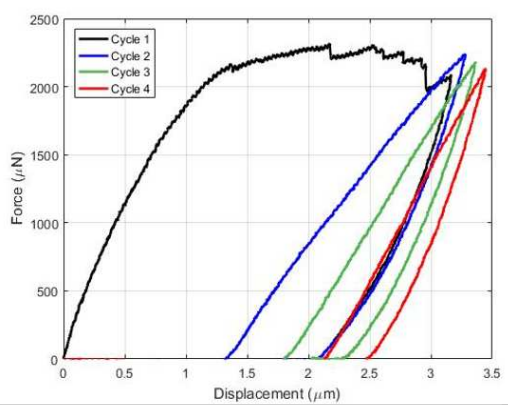

(b)

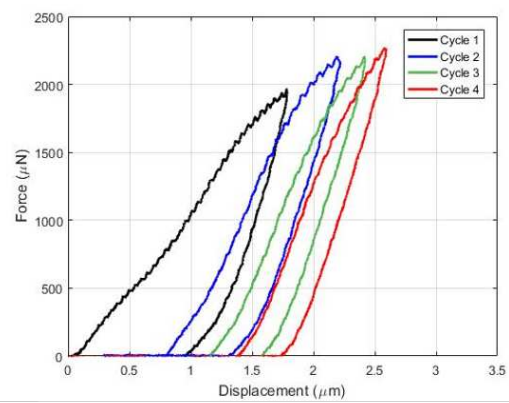

(c)

Figure 3: Force-vs-displacements plots for repeated loading-unloading cycles.

contact during activation of the bistable mechanism.

Several lattices with the geometry shown in Fig. 2 (a) have been fabricated by the multiphoton lithography (MPL) technique, which permit to obtain beam members with cross sections of $250 \mathrm{~nm}$ minimum radius (Fig. 2, b). Although the fabricated lattices have beam members which are rigidly connected to each other at the nodes, these lattices can still inherit the bistable behavior from the parent tensegrity architecture, when beam bending, shear, and torsion remain limited, as we will show in the next section.

The material for the specimens has been prepared by mixing organic-inorganic constituents, such as: $\mathrm{Zr}$ -

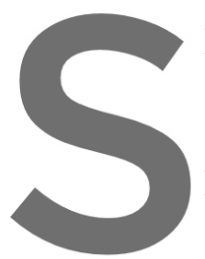

DMAEMA composed o

(DMAEMA) (Sigma-A

3-(trimethoxysilyl)prop

hydrochloric acid into a

(IV) propoxide solution
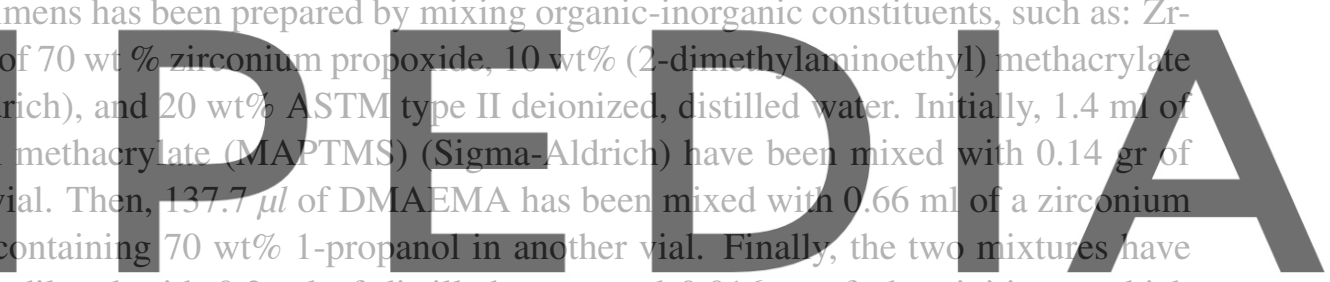

been mixed together and diluted with $0.2 \mathrm{ml}$ of distilled water and $0.016 \mathrm{gr}$ of photoinitiator, which

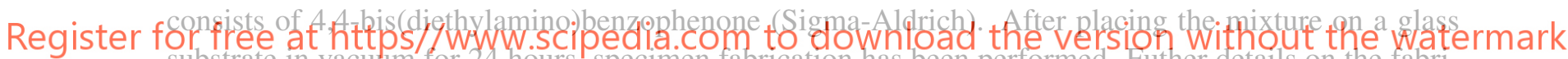
substrate in vaculum lor 24 hours, specimen fabrication has been performed. Futher details on the fabri-

cation procedure are reported in [22]. The Young modulus of the fabricated material has been estimated with preliminary tests to be equal to $E=1.281 \mathrm{GPa}$.

Indentation experiment have been performed by applying unilateral displacement-controlled loadingunloading cycles of increasing amplitude (Fig. 2, c). Results of the testing are shown in Fig. 3. We can observe that force-vs-displacement curves have the following common features: there is a marked softening behavior; the softening part of the curve leads to a secondary stable equilibrium configuration upon unloading; the secondary configuration is preserved during successive loading-unloading cycles; the curve presents a slight visocelastic character. Moreover, it can be oserved that, in association with the softening behavior, the three middle triangles undergo a twisting motion. In addition, the curves show that when the loads is close to reaching the peak value, there can be microcracking events taking place, which are denoted by a sawtooth pattern in the curve. 


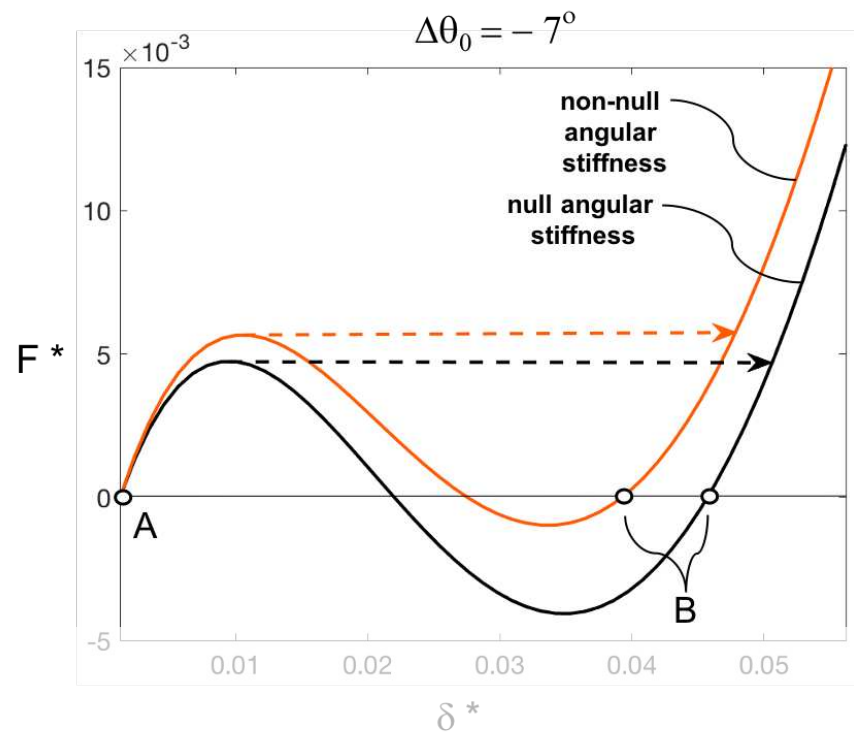

Figure 4: Dimensionless force-vs-displacement plot obtained numerically for the bistable assembly.

4 NUMERICAL EXPERIMENTS

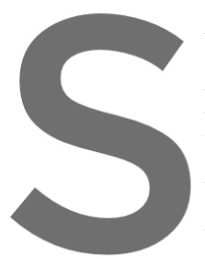

We implemented a reduce proach (see, e.g. [26]-[28 while members can only

they are rigid with respect to tic springs are placed at th
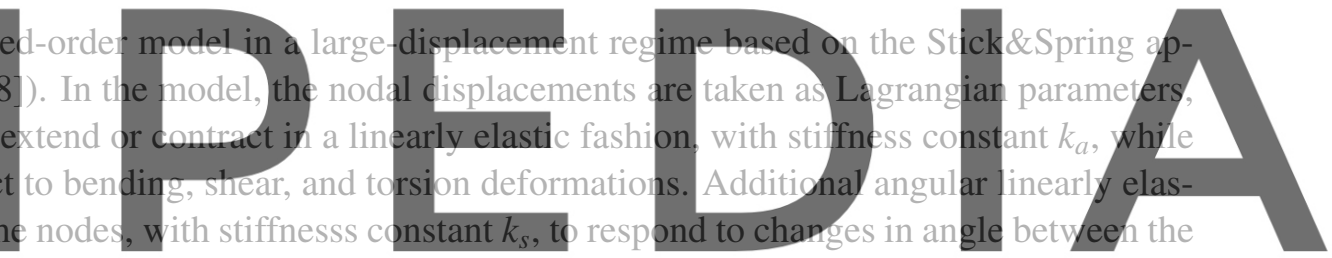

axes of pairs of members which are not part of a triangle. These angular springs account for the actual

Register for free af

Figure 4 shows the static response to a vertical downward load applied to the studied assembly. In the plot, the dimensionless load $F^{*}$ is one third of the actual load divided by the stiffness constant of the shortest beam and by the beams' minimum diameter, while the dimensionless displacement $\delta$ is the vertical displacement divided by the height of the assembly. As to the geometric parameters in this simulation, we have that $a / b=0.7, a / h=0.25, \Delta \theta_{0}=-7$ degrees, where $a$ and $b$ are the circumscribed radii to the bottom and top bases of the unit, $h$ is the height of the assembly, and $\Delta \theta_{0}=\theta_{0}-\pi / 6$. The two curves differ in the value of the angular stiffness constant, this is null for the black curve, and it satisfies $k_{s} /\left(a^{2} k_{a}\right)=0.00025$ for the color (grey) curve. The simulation is consistent with the fact that in a force-controlled loading-unloading experiment, the system would snap from a primary stable configuration (corresponding to point $\mathrm{A}$ in Fig. 4) to a secondary stable configuration (corresponding to points B in Fig. 4), passing along a softening-type path before snapping. A behavior which is qualitatively similar to that observed in the indentation experiments, while the differences can be partly ascribed to the viscoleastic behavior of the photoresistive material.

We then performed the same numerical experiment on a three-layer assembly obtained by superposing copies of the previously considered system on top of each other (Fig. 5, a). The three layers differ from 


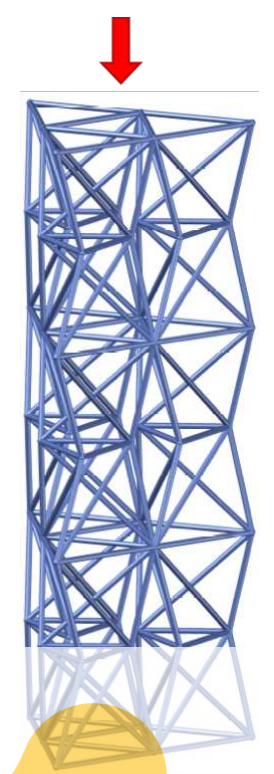

(a)

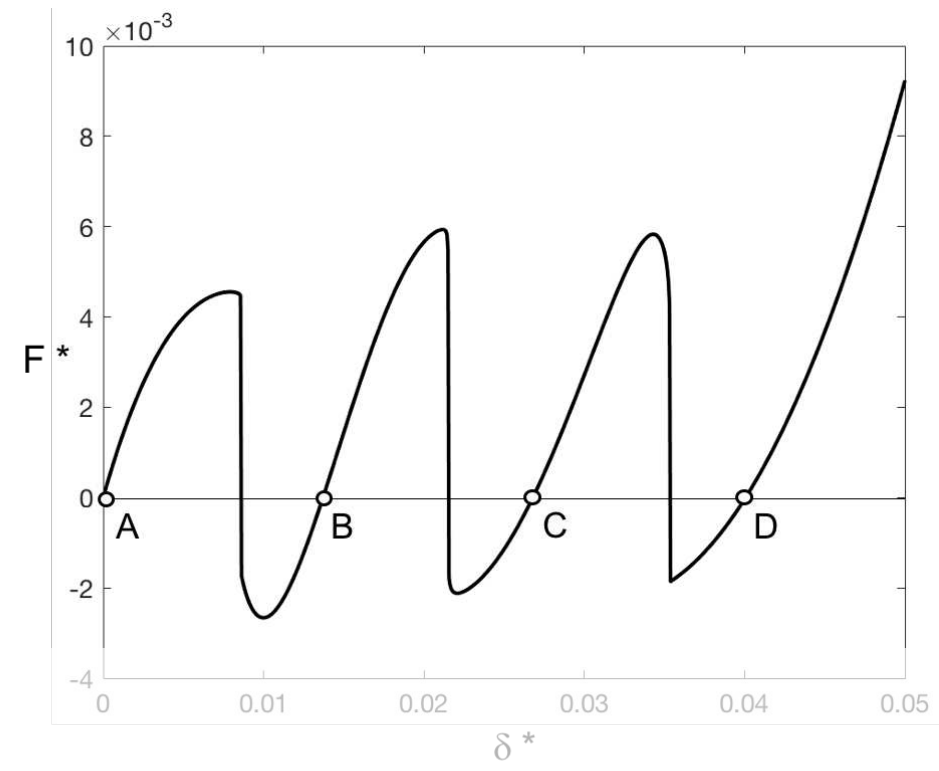

(b)

Figure 5: (a) Three-layer assembly. (b) Dimensionless force-vs-displacement plot of the three-layer assembly.

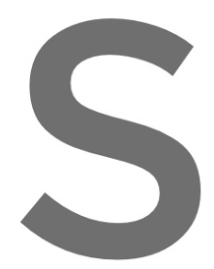

one another in the stiffines

one layer to another in $\phi$

same as in the previous ast

displacement curve (Fi

Fig. 5, b), related to the bi
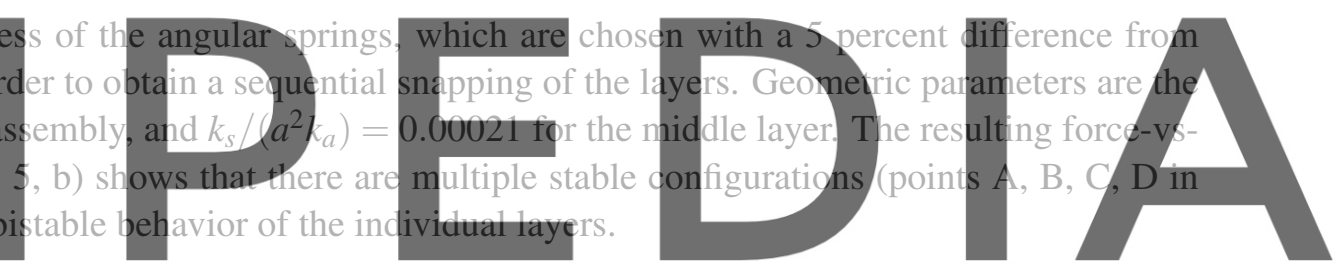

\section{Register for freNCLU hitens/www.scipedia.com to download the version without the watermark}

- A method to obtain bistable lattices from parent tensegrity architectures has been illustrated.

- A bistable assembly composed of triangular tensegrity prisms has been designed, fabricated by MPL, and subjected to indentation testing.

- The experimental force-vs-displacement curves highlight a softening response which brings the structure to a secondary stable equilibrium configuration. Such secondary configuration is maintained during successive loading-unloading cycles. In addition, a slight viscoelastic behavior is observed, together with occasional microcraking events.

- A Stick\&Spring reduced-order model is employed to simulate indentation esperiment, confirming that the observed experimental behavior results from the bistable design of the constituent units.

- A three-layer system is analyzed numerically to show that multistable lattices can be obtained. 


\section{Acknowledgements}

This research is partially supported by the National Science Foundation (NSF) under the Scalable 475 Nanomanufacturing (SNM) Program, grant number 1449305. A.M. acknowledges financial support from the Italian Ministry of Education, University, and Research (MIUR) under the PRIN 2017 National Grant '3D printing: a bridge to the future' (grant number 2017L7X3CS 004). N.S. and F.F. acknowledge financial support from MIUR under the PRIN 2017 National Grant 'Multiscale Innovative Materials and Structures'(grant number 2017J4EAYB).

\section{REFERENCES}

[1] Liu, Z., Zhang, X., Mao, Y., Zhu, Y.Y., Yang, Z., Chan, C.T. and Sheng, P. Locally resonant sonic materials. Science (2000) 289:1734-1736.

[2] Lu, M.H., Feng, L., Chen, Y.F. Phononic crystals and acoustic metamaterials. Mater. Today (2009) 12:34-42.

[3] Maldovan, M., Sound and heat revolution in phononics. Nature (2013) 503:209-217.

[4] Brunet, T., Leng, J., Mondain-Monva, O. Soft acoustic metamaterials. Science (2013) 342:323-324.

[5] Deng B., Mo C., Tournat V., Bertoldi K., Raney J.R. Focusing and Mode Separation of Elastic Vector Solitons in a 2D Soft Mechanical Metamaterial, Phys. Rev. Lett. (2019) 123:024101.
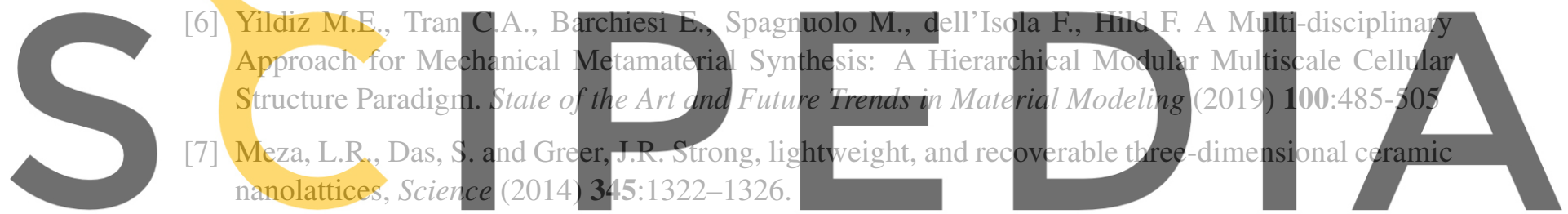

[8] Zheng, X. et al., Ultralight, ultrastiff mechanical metamaterials, Science (2014) 344:6190.

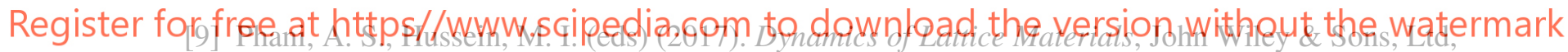
Chichester, UK.

[10] F. Fraternali, L. Senatore, C. Daraio, Solitary waves on tensegrity lattices. Journal of the Mechanics and Physics of Solids (2012) 60:1137-1144.

[11] F. Fraternali, G. Carpentieri, A. Amendola, R.E. Skelton, V.F. Nesterenko, Multiscale tunability of solitary wave dynamics in tensegrity metamaterials. Applied Physics Letters. (2014) 105:201903.

[12] C. Davini, A. Micheletti, P. Podio-Guidugli, On the impulsive dynamics of T3 tensegrity chains, Meccanica (2016) 51:2763-2776.

[13] A. Micheletti, G. Ruscica, F. Fraternali, On the compact wave dynamics of tensegrity beams in multiple dimensions. Nonlinear Dynamics (2019) 98:2737-2753.

[14] Shan, S., Kang, S.H., Raney, J.R., Wang, P., Fang, L., Candido, F., Lewis, J.A., Bertoldi, K. Multistable Architected Materials for Trapping Elastic Strain Energy. Adv. Mater.(2015) 27:4296-4301.

[15] Raney, J.R., Nadkarni, N., Daraio, C., Kochmann, D.M., Lewis, J.A., Bertoldi K. Stable propagation of mechanical signals in soft media using stored elastic energy, PNAS (2016) 113:9722-9727. 
[16] Osama, R.B., André, F., Chiara, D. Switching and cascading elastic vibrations Proceedings of the National Academy of Sciences (2017) 114:4603-4606.

[17] Chen T., Bilal O.R., Shea K., Daraio C. Harnessing bistability for directional propulsion of soft, untethered robots Proceedings of the National Academy of Sciences (2018) 115:5698-5702.

[18] Deng B., Wang P., Tournat V., Bertoldi K. Nonlinear transition waves in free-standing bistable chains, Journal of the Mechanics and Physics of Solids (2019) pp. 103661

[19] Jeong, H.Y., An, S., Seo, I.C. et al. 3D printing of twisting and rotational bistable structures with tuning elements. Sci Rep (2019) 9324.

[20] Oppenheim I.J.,Williams W.O. Geometric effects in an elastic tensegrity structure. J. Elast. (2000) 59, pp. 51-65.

[21] Micheletti A. Bistable regimes in an elastic tensegrity system, Proc. R. Soc. A (2013) 469.

[22] Ovsianikov A., Viertl J., Chichkov B., Oubaha M., MacCraith B., Sakellari I., Giakoumaki A., Gray D., Vamvakaki M., Farsari M., Fotakis C. Ultra-low shrinkage hybrid photosensitive material for two-photon polymerization microfabrication. ACS Nano (2008) 2:2257-2262.

[23] Sakellari I., Kabouraki E., Gray D., Purlys V., Fotakis C., Pikulin A., Bityurin N., Vamvakaki M., Farsari M. Diffusion-Assisted High-Resolution Direct Femtosecond Laser Writing. ACS Nano (2012) 6:2302-2311.

[24] Calladine, C.R., Pellegrino, S. First Order Infinitesimal Mechanisms, Int. J. Sol. Struct. (1991)

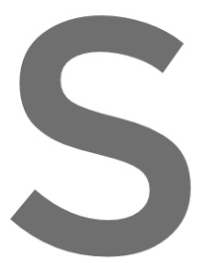
27:505-515,

[25] C.R. Calladine, Buckminster Construction of Stiff Frames,

[26] A. Favata, A. Micheletti, P. Podio-Guidugli, Spring Structures, International Journal of Er

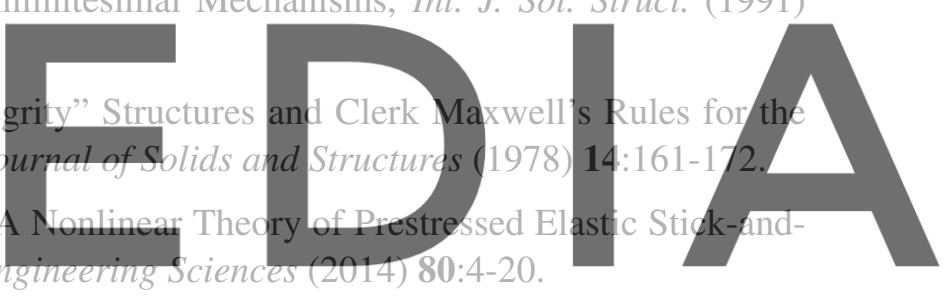

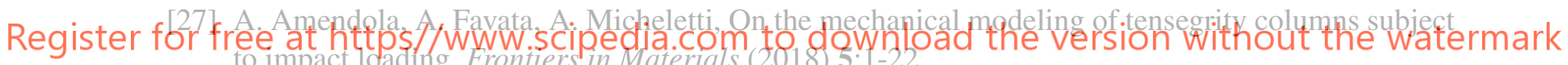

[28] Favata, A., Micheletti, A., Podio-Guidugli, P., Pugno, N.M., How graphene flexes and stretches under concomitant bending couples and tractions. Meccanica (2017) 52:1601-1624. 